\title{
Animal trait variation at the within-individual level: Erythrocyte size variation and malaria infection in a tropical lizard
}

\author{
Virnaliz Cruz ${ }^{\text {Corresp., } 1,2}{ }^{2}$, Omar Cruz-Pantoja ${ }^{3}$, Raymond Tremblay ${ }^{4}$, Miguel Acevedo ${ }^{1}$ \\ ${ }^{1}$ Department of Wildlife Ecology and Conservation, University of Florida, Gainesville, Florida, United States \\ 2 Department of Environmental Science, Universidad de Puerto Rico, Rio Pidras, Puerto Rico, United States \\ 3 Department of Computer Science, Universidad de Puerto Rico, Recinto de Rio Pidras, San Juan, Puerto Rico, United States \\ 4 Department of Biology, Universidad de Puerto Rico, Humacao, Puerto Rico, United States \\ Corresponding Author: Virnaliz Cruz \\ Email address: virnaliz.cruz@weecology.org
}

High levels of within-individual variation (WIV) in reiterative components in plants such as leaves, flowers, and fruits have been shown to increase individual fitness by multiple mechanisms including mediating interactions with natural enemies. This relationship between WIV and fitness has been studied almost exclusively in plant systems. While animals do not exhibit conspicuous reiterative components, they have traits that can vary at the individual level such as erythrocyte size. It is currently unknown if WIV in animals can influence individual fitness by mediating the outcome of interactions with natural enemies as it has been shown in plants. To address this issue, we tested for a relationship between WIV in erythrocyte size, hemoparasite infection status, and body condition (a proxy for fitness) in a Caribbean anole lizard. We quantified the coefficient of variation of adult erythrocytes size in $\$ n=95 \$$ infected and $\$ n=107 \$$ non-infected lizards. We found higher degrees of erythrocyte size variation in infected lizards than in non-infected individuals. However, we found no significant relationship between infection status or erythrocyte size variation, and lizard body condition. These results suggest that higher WIV in erythrocyte size in infected lizards is not necessarily adaptive but likely a consequence of the host response to infection. Many hemoparasites destroy their host cells as part of their life cycle. To compensate, the host lizard may respond by increasing production of erythrocytes resulting in higher WIV. Our results emphasize the need to better understand the role of within-animal variation as a neglected driver or consequence of ecological and evolutionary interactions. 


\title{
Animal trait variation at the within-individual level: Erythrocyte size variation and malaria infection in a tropical lizard
}

\author{
Virnaliz Cruz ${ }^{1,4}$, Omar Cruz-Pantoja ${ }^{2}$, Raymond L. Tremblay ${ }^{3}$, and Miguel A. \\ Acevedo $^{4}$

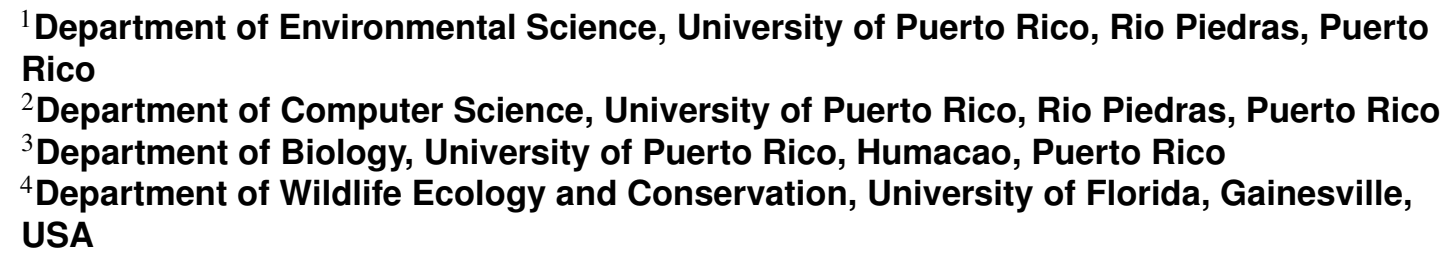

\begin{abstract}
High levels of within-individual variation (WIV) in reiterative components in plants such as leaves, flowers, and fruits have been shown to increase individual fitness by multiple mechanisms including mediating interactions with natural enemies. This relationship between WIV and fitness has been studied almost exclusively in plant systems. While animals do not exhibit conspicuous reiterative components, they have traits that can vary at the individual level such as erythrocyte size. It is currently unknown if WIV in animals can influence individual fitness by mediating the outcome of interactions with natural enemies as it has been shown in plants. To address this issue, we tested for a relationship between WIV in erythrocyte size, hemoparasite infection status, and body condition (a proxy for fitness) in a Caribbean anole lizard. We quantified the coefficient of variation of adult erythrocytes size in $n=95$ infected and $n=107$ non-infected lizards. We found higher degrees of erythrocyte size variation in infected lizards than in non-infected individuals. However, we found no significant relationship between infection status or erythrocyte size variation, and lizard body condition. These results suggest that higher WIV in erythrocyte size in infected lizards is not necessarily adaptive but likely a consequence of the host response to infection. Many hemoparasites destroy their host cells as part of their life cycle. To compensate, the host lizard may respond by increasing production of erythrocytes resulting in higher WIV. Our results emphasize the need to better understand the role of within-animal variation as a neglected driver or consequence of ecological and evolutionary interactions.
\end{abstract}

\section{INTRODUCTION}

Phenotypic traits vary among individuals of the same species. For example, the amount of nectar production (Real and Rathcke, 1991), the density of mycorrhizal fungi on plant roots (Stahl et al., 1990), and the lethality of venom in animals (Castro et al., 2013) vary among individuals. For centuries, this among-individual variation has been recognized as the building block of natural selection. In contrast, we know little about the eco-evolutionary consequences of within-individual variation (WIV). Until recently, WIV was deemed as a mere statistical nuisance that could be rectified with appropriate experimental design practices and statistical techniques (Herrera, 2009). However, recent studies emphasize the role of WIV in phenotypic traits of plants as a mediator of individual fitness (Austen et al., 2015; Shimada et al., 2015; Herrera et al., 2015).

Plant fitness has been shown to improve with increasing WIV by mediating ecological interactions with co-evolved symbionts or natural enemies. For example, in the presence of higher degrees of withinplant variation in nectar volume pollinators may limit the number of flowers they visit to avoid the risk 
of not encountering nectar in the next visited flower (Shafir, 2000). This risk aversion might improve plant fitness by reducing geitonogamy, a type of self-pollination (Biernaskie et al., 2002). Similarly, WIV is hypothesized to grant plants a mosaic of protection against natural enemies such as insect herbivores (Whitham, 1983). While insects often have short generations that promote fast adaptation, WIV limits their ability to adapt simultaneously to the spatially complex mosaic of reiterative components (Whitham and Slobodchikoff, 1981). For example, when cotton plants are exposed to leaf damage by the moth Spodoptera littoralis, young top leaves produce more secondary metabolites than older leaves or young side shoots. This variation in response to herbivory by young and old leaves results in a spatial mosaic of secondary metabolites that increase plant fitness by disincentivizing leaf consumption (Anderson and Agrell, 2005). Similarly, mountain birch, Betula pubescens ssp. tortuosa, produces a damage-induced mosaic of resistance by varying the number of secondary metabolites in leaves. When Oporinia autumnata moths were reared using leaves that were adjacent to the mechanically damaged leaves, the larvae showed retarded growth (Haukioja and Niemelä, 1979). With increasing supporting evidence from empirical studies, WIV has been proposed as a key mediator of natural enemies interactions resulting in improved fitness in many plant systems.

This hypothesized relationship between WIV, natural enemies, and individual fitness remains unexplored in animal systems. Animals are not necessarily characterized for having external conspicuous reiterative traits like leaves, flowers, or fruits in plants. Still, vertebrates can exhibit within-individual temporal variation in behavioral traits such as movement activity, sexual displays, or vocalizations (Nakayama et al., 2016; Tanner and Bee, 2019; Bee, 2004). Animals could also exhibit WIV in internal components such as cells size. For instance, Price-Jones (1929), in one of the first and few studies on WIV in non-plant systems, found that humans that suffered from pernicious anemia had higher rates of WIV in the diameter of their erythrocytes compared to healthy individuals. Erythrocyte size variability returned to pre-disease levels after individuals were treated for anemia suggesting that WIV resulted from the disease. In this example, the disease mediated WIV in erythrocyte size. Alternatively, variation in erythrocyte traits can mediate the likelihood of disease infection. For instance, the sickle cell gene is responsible for erythrocyte structural and functional changes. Humans that are heterozygous for the sickle cell gene can still become infected with malaria but have a selective advantage (Allison, 1954). The improved fitness of these heterozygous individuals is explained by variation in sickling rates of Plasmodium falciparum infected and non-infected erythrocytes. The sickling rate of non-infected cells is two to eight times lower (Roth et al., 1978). These results emphasize the role of erythrocytes as reiterative components and hypothesize how a mosaic of erythrocyte sizes can mediate the probability of infection or, alternatively, be the result of disease.

Confirmation of an adaptive role of increased WIV protection against parasite infection would require three types of evidence. First, parasite infection should result in a decrease in host fitness. This could be quantified as a decrease in reproductive output, survival, or sub-lethal effects such as a decrease in body condition (Acevedo et al., 2019). Second, there should be evidence of a difference in the level of WIV between infected and non-infected individuals. Third, individuals with higher degrees of WIV will have higher fitness in comparison with those with lower WIV. Alternatively, if there is evidence of a relationship between infection and WIV without evidence of a relationship with host fitness, it could mean that changes in WIV are a proximate consequence of infection with no adaptive consequence.

To address this issue, we tested for a relationship between WIV in erythrocyte size and Plasmodium azurophilum infection in Anolis gundlachi-a lizard host in Puerto Rico. Specifically, we ask: (1) Do Plasmodium infected lizards have lower body condition? (2) Is infection status related to WIV in erythrocyte size? (3) Is body condition better on individuals with higher WIV in erythrocyte size? This host-parasite system is appropriate to test for this relationship for multiple reasons. First, while $P$. azurophilum is a common parasite of anoles in the Caribbean, in Puerto Rico $\sim 90 \%$ of infections occur on A. gundlachi. This level of specialization and other empirical evidence suggests that this host-parasite system has co-evolved for many years (Schall, 1990; Schall et al., 2000). Second, the host lives in high abundances (2000 ind/ha; (Reagan and Waide, 1996)) allowing for the large sample sizes required to properly quantify WIV. Third, the population that we studied is protected from human intervention, which helps control for anthropogenetic pressures. 


\section{METHODS}

\section{Study site and species}

To answer these questions our data consists of a subset of individuals from an ongoing long-term study on the dynamics of the Anolis-Plasmodium host-parasite system at El Verde Field Station, in the Luquillo

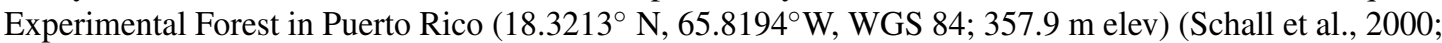
Otero et al., 2019). The surveys took place during the summer season of 2015 and the winter season of 2016. The Institutional Animal Care and Use Committee protocols from the University of Puerto Rico provided the permits for the handling of lizards, IACUC (01005-01-09-2015).

Out of the seven species of anoles inhabiting El Verde, we selected Anolis gundlachi as our study species because it is the member of the anole community with the highest prevalence of Plasmodium parasite infection in the site. Between 2015 and 2017 the probability of infections was $0.10-0.19$ for males, and $0.06-0.12$ for females (Otero et al., 2019). This anole is a medium-sized montane lizard, part of the trunk-ground ecomorph, with a snout-vent length (SVL) range of $42-72 \mathrm{~mm}$ (Reagan and Waide, 1996). This species can be infected by three Plasmodium species: P. azurophilum, P. floridense, and $P$. leucocytica. We analyzed infections by $P$. azurophilum because this is the most common blood parasite at the site, accounting for $60-80 \%$ of infections (Schall et al., 2000; Otero et al., 2019). Also, $P$. azurophilum is the only member of this Plasmodium community known to destroy lizard erythrocytes (Schall, 1996). Co-infections are infrequent. Still, we restricted our analysis to single infected individuals to ensure our results were not biased by potential interacting effects of multiple blood parasites (see below).

We captured anoles by hand or using the lasso technique while the lizards were perching on tree trunks, branches, or on the ground. The lizards were briefly kept in individual bags and moved to the laboratory where they were processed to identify sex, measure the snout-to-vent length (SVL) and weight, and obtain a blood sample via toe clip (Schall and Vogt, 1993). We used this blood sampling method because it provides enough blood to accurately diagnose infection by microscopy and PCR (see below). Also, toe clipping provides a permanent mark that prevents sampling of an individual more than once. Elevated levels of corticosterone have been shown to alter the hematopoietic cell composition and immune response in other animals; however, this method has less impact on corticosterone levels than implanted Passive Integrated Transponder (PIT) tags (Langkilde and Shine, 2006). All lizards were released back to the general area in which they were found within $24 \mathrm{hrs}$.

\section{Infection diagnostics}

We diagnosed Plasmodium infection using microscopy and verifying through polymerase chain reaction (PCR). Combining both methods assures the most accurate classification of infection status. For microscopy diagnosis, we made thin-blood smears on glass slides. The thin blood smears were fixed with $100 \%$ methanol for a minute and stained with Giemsa at a $\mathrm{pH}$ of 7.5 for 50 minutes. We examined the blood smears at 1000x magnification using a Nikon Eclipse E2000 light microscope for 6 - 10 minutes (Otero et al., 2019). We classified the Plasmodium species detected by morphological traits (Telford, 2009). We considered a lizard infected if we detected through microscopy at least one cell invaded by P. azurophilum, and if PCR confirmed infection by positively amplifying Plasmodium. Similarly, we considered a lizard non-infected if we could not visually detect an invaded cell using microscopy and the PCR did not amplify Plasmodium. To ensure the most accurate classification of infection status, if there was a mismatch between the slide blood smear and the PCR result the individual was removed from the analysis.

Drops of lizard blood were spotted on Whatman filter paper and stored at $-20{ }^{\circ} \mathrm{C}$ in sealed bags with silica gel for further PCR testing. DNA was extracted from blood-spot filters using the DNeasy Extraction kit with some modifications to the manufacturer's instructions. To extract the blood from the filter paper, $180 \mu \mathrm{L}$ of Buffer ATL and $20 \mu \mathrm{L}$ Protease K was added. The samples were mixed and incubated at $56^{\circ} \mathrm{C}$ overnight with shaking. The next day we added $200 \mu \mathrm{L}$ of Buffer AL to the sample, mixed by thoroughly shaking for 15 seconds, and incubated at $56^{\circ} \mathrm{C}$ for $15 \mathrm{~min}$. Then, $200 \mu \mathrm{L}$ ethanol was added to each sample. To elute DNA, we added $50 \mu \mathrm{L}$ to $75 \mu \mathrm{L}$ Buffer AE to each sample and incubated it for 5 minutes at room temperature. The extracted DNA (eluate) was stored at $-20{ }^{\circ} \mathrm{C}$. A partial fragment of the mitochondrial cytochrome b gene was amplified using nested PCR (nPCR). The nPCR was done with Taq PCR Master Mix kit (Qiagen cat 201445) under the conditions described by Perkins and Schall (2002) with minor modifications. Briefly, an outer reaction using primers DW2 F 5'- TAA TGC CTA GAC GTA 
TTC CTG ATT ATC CAG - 3' DW4 R 5'- TGT TTG CTT GGG AGC TGT AAT CAT AAT GTG 3 'and $2 \mu \mathrm{L}$ of genomic DNA was subject to thermal cycling program included an initial denaturation step at $94^{\circ} \mathrm{C}$ for $4 \mathrm{~min}$ followed by 35 cycles of $94{ }^{\circ} \mathrm{C}$ for 20 seconds, $60{ }^{\circ} \mathrm{C}$ for 30 seconds, and $72^{\circ} \mathrm{C}$ for 1.5 minutes. The nested reactions were done with $1 \mu \mathrm{L}$ of the previous PCR product and primers DW1 5'-TCA ACA ATG ACT TTA TTT GG-3' and DW6 5'-GGG AGC TGT AAT CAT AAT GTG-3' under initial denaturation step at $94^{\circ} \mathrm{C}$ for 1 min followed by 40 cycles of $94^{\circ} \mathrm{C}$ for 20 seconds, $50^{\circ} \mathrm{C}$ for 20 seconds, and $72^{\circ} \mathrm{C}$ for 1,5 minutes and then $72{ }^{\circ} \mathrm{C}$ for 7 minutes. Positive and negative reaction controls were included. Amplification products were resolved in a $2 \%$ agarose gel stained with gelStarTM (Lonza, cat \# 50535) and visualized under ultraviolet light. The primers only amplified the malarial parasite DNA. PCR complemented diagnosis by helping avoid false negatives resulting from individuals with low parasitemia that may be missed by the slides.

\section{Erythrocyte image capturing and measurement}

We photographed 10 microscope optical fields for each lizard's thin blood smear (Fig. 1). Optical fields were selected at random. Fields in which less than 10 mature erythrocytes were measurable were discarded. We measured only mature erythrocytes in each picture and disregarded other hematocytes because we wanted to estimate variation in erythrocyte size independent of stage. We took the pictures at 40x power using a Nikon DS-Fi2 camera head and a Nikon DS-U3 microscope camera controller. This setting provided high-quality pictures that allowed precise measurements of the erythrocyte area. Since measuring erythrocyte size in clumps of cells is challenging and may result in inaccurate measures, we considered cells to be measurable if there was at least $0.070 \mu \mathrm{m}$ of space between cells, and if they were not damaged during the smear making process. We also discriminated between infected and non-infected erythrocytes. We discarded 19 Plasmodium-invaded cells (out of a total of 50,241 measured erythrocytes) from the analysis because erythrocyte sickling rate has been observed to differ between Plasmodium stages (Roth et al., 1978). By restricting our analysis on mature erythrocytes that were not invaded by the parasite we segregated the processes allowing us to assess the global effects of Plasmodium on WIV independent of local effects.

We measured the area of each erythrocyte in each optical field with the image processing software Image J (Schneider et al., 2012). We set the scale to $50.0 \mu \mathrm{m}$ and the image type to 8-bit grayscale to allow segmentation by pixel color intensity, also known as image thresholding. The image threshold was manually adjusted for each image due to differences in the sharpness, quality of staining, and brightness of images. We selected the optimum threshold that allowed us to minimize measurement error by decreasing the amount of whitespace or non-erythrocyte area inside the measurement polygon. We stored measurements of erythrocyte per picture automatically on individual spreadsheets with their unique ID as the file name. We also took lower resolution pictures of the selected erythrocytes per picture which had the order in which erythrocytes were measured. This allowed us to keep a permanent record of which measurement belonged to which erythrocyte for verification purposes while maintaining our original pictures intact to ensure study reproducibility.

\section{Data analyses}

We conducted a prospective power analysis to estimate an appropriate sample size to test our hypotheses with statistical power $>0.8$. We analyzed pilot data $(n=21)$ to estimate the variance and mean coefficient of variation of the population. To estimate the effect size, we used Cohen's $d$ defined as, $d=\frac{\bar{x}_{\text {inf }}-\bar{x}_{\text {ninf }}}{S D_{\text {pooled }}}$, where $\bar{x}_{\text {inf }}=12.59$ (infected CV) and $\bar{x}_{\text {ninf }}=12.02$ (non-infected CV). We set a significance level of $\alpha=$ 0.05 and a power of 0.8 as it is conventionally done in studies of ecology and evolution.

We quantified within-individual erythrocyte size variation of $A$. gundlachi using the coefficient of variation $(C V)$, such that:

$$
C V_{i}=\frac{s_{i}}{\bar{x}_{i}} \times 100
$$

where $\bar{x}_{i}$ represents the sample mean and $s_{i}$ the sample standard deviation of the size of mature erythrocytes of lizard $i$. The coefficient of variation $(\mathrm{CV})$ is a measure of relative variation allowing comparison among lizards while controlling for mean erythrocyte size (Donnelly and Kramer, 1999). This measure is commonly used in similar studies to quantify within-individual variation (Biernaskie et al., 2002; Herrera et al., 2015; Langkilde and Shine, 2006). 
We modeled lizard body condition index (BCI) as a function of infection status, and sex and season as controlling variables using a multiple linear regression. Although reproduction data would be ideal to test for the negative consequences of infection on individual fitness, collecting breeding data is challenging and often infeasible in many natural systems including anoles (Jakob et al., 1996). Here we use BCI as a proxy for fitness as the next best alternative. We calculated BCI as the residuals of the linear regression of $\log 10$ weight (g) explained by $\log 10$ lizard SVL (mm) (Cox and Calsbeek, 2015). Positive residuals suggest a better body condition relative to the population average (Schall et al., 2000). Male A.gundlachi $\mathrm{BCI}$ has been shown to be higher than females and $\mathrm{BCI}$ also responds to seasonal variation in resource availability (Schall et al., 2000). Previous studies validate the use of BCI to represent the energetic state of animals (Ardia, 2005; Schulte-Hostedde et al., 2005). Still, a study found that BCI is weakly related to survival in Anolis sagrei (Cox and Calsbeek, 2015) and a meta-analysis found small, negative average effect sizes between BCI and parasite infection that was stronger in laboratory settings (Sánchez et al., 2018). In an experimental setting, mass/SVL was found to be the most accurate estimator for the mass of chemically extracted fat for a reptile among five compared estimators (Sion et al., 2021). Therefore, while $\mathrm{BCI}$ is the best approximation to fitness given our data constraints we are careful interpreting the results given its mixed support as a proxy for fitness (Wilder et al., 2016).

To test for a relationship between $P$. azurophilum infection and WIV in erythrocyte size, we modeled infection status as a function of CV. Previous studies show that larger males in the summer season have a higher probability of getting infected (Otero et al., 2019). Therefore, we included sex, season, and body size (SVL) as controlling covaraites. We modeled this relationship using a generalized linear model with a Bernoulli distribution and logit link function.

To test if individuals with higher WIV have improved fitness, we modeled BCI as a function of CV in erythrocyte size with sex and season as controlling covaraites using a multiple linear regression. These controlling covariates were included because previous studies in this system found that body condition is higher in males and during the summer season where food resources for lizards are more abundant (Schall et al., 2000).

\section{RESULTS}

In the power analysis to determine appropriate sample size, infected individuals' erythrocyte size was more variable ( $\mathrm{CV}=12.59 \pm 1.74 \mathrm{SD})$ than non-infected individual erythrocyte size $(\mathrm{CV}$ of $12.02 \pm 1.27$ $\mathrm{SD})$. With an estimated effect size of $d=0.38$, the power analysis suggested an optimal sample size of $N=112$ individuals in each category (infected and non-infected). This sample size was a conservative estimate that falls under the small-medium effect size category ( 0.20 standard deviation difference is considered small, and 0.50 standard deviation difference is considered medium) (Cohen, 1992). Our final data set allowed us to analyze a total $N_{\text {infected }}=95$ and $N_{\text {noninfected }}=107$ individuals after discarding individuals with co-infections or poorly stained slides. Therefore, our overall sample size was appropriate to attain appropriate power.

A linear model predicted no significant relationship between infection status and BCI ( $\beta_{\text {infected }}=$ $0.015 \pm 0.01$ SE, $P=0.14$; Fig. 2). Similarly, this model predicted no significant effect of season on BCI $\left(\beta_{\text {winter }}=-0.02 \pm 0.01 \mathrm{SE}, P=0.05\right)$. Still, the model predicted that males would have higher body condition than females $\left(\beta_{\text {males }}=0.03 \pm 0.01, P=0.01\right)$.

We found that the $\mathrm{CV}$ of erythrocyte size is a strong predictor of infection status $(z=2.92, P=0.003$, Fig. 3). The binomial model predicted that the probability of being infected increases $1.43(1.13-1.83$ 95\% CI) times with a unit increase in $\mathrm{CV}$. The model also predicted that the probability of being infected is $0.63(0.34-1.1695 \% \mathrm{CI})$ times in the winter compared to the summer, but this relationship is uncertain $(z=-1.48, P=0.14)$. Similarly, the model predicted that the probability of being infected is 0.79 $(0.33-1.995 \% \mathrm{CI})$ times in males when compared to females, but this relationship was also uncertain $(z=-0.52, P=0.60)$. The probability of infection is predicted to increase $1.08(1.03-1.1295 \% \mathrm{CI})$ times with a mm increase in SVL $(z=3.3, P<0.001$; Fig. 3).

While we found $\mathrm{CV}$ of erythrocyte size to be a key predictor for the probability of infection, $\mathrm{CV}$ was not a significant predictor of BCI $(\beta=-0.001 \pm 0.004 \mathrm{SE}, P=0.86$; Fig. 4). Similar to the analysis presented above on BCI predicted by infection status, season was not a significant predictor of $\mathrm{BCI}\left(\beta_{\text {winter }}=-0.02 \pm 0.01 \mathrm{SE}, P=0.08\right)$, but males had better body condition than females $\left(\beta_{\text {males }}=\right.$ $0.03 \pm 0.01 \mathrm{SE}, P=0.006)$ 


\section{DISCUSSION}

Studies in plant systems show that WIV increases individual fitness through multiple mechanisms including mediating the outcome of interactions with natural enemies. Testing this hypothesis in animal systems requires studying reiterative structures that can be exposed to selective processes. Here we studied the relationship between WIV in erythrocyte size, hemoparasite infection, and body condition in the Caribbean lizard Anolis gundlachi. Our results show that infected individuals had a higher WIV in erythrocyte size than non-infected individuals, but WIV was not related to the host body condition. This result suggests that the higher WIV in infected may not necessarily be adaptive but likely a consequence of infection.

There are three potential explanations for the lack of variation in BCI due to infection status or WIV in erythrocyte size. First, virulence - the negative consequences of infection for host fitness-may be low or undetectable in this system. Virulence varies widely in lizard malaria systems. For example, $P$. mexicanum infection on Sceloporus occidentalis in California, USA and P. agamae infection on Agama agama in Sierra Leone, West Africa results in lower hemoglobin levels and a decreased in physiological characteristics such as oxygen consumption and stamina (Schall, 1990). In contrast, there are few indications of virulence to Caribbean hosts. For instance, Anolis sabanus infected by $P$. azurophilum had no difference in body temperature, broken tails, habitat use, or intraspecific interactions with non-infected individuals (Schall and Staats, 2002). Similarly, other studies have found little evidence of decreases in body condition due to infection in Caribbean lizards (Pearson, 2000; Otero et al., 2019). This variation in virulence is also present in Plasmodium infection to avian hosts. For example, Plasmodium infection is linked to a dramatic decrease in Hawaiian honeycreeper population size (Atkinson et al., 1995). Still, chronic infections show no important negative consequences for American songbirds (Matthews et al., 2016). Second, our results show that infected individuals have, on average, better body condition, but the uncertainty is too large to make this pattern statistically significant. This result could also mean that $A$. gundlachi is slightly tolerant of P. azurophilum infection, but the effect size is too small to be easily detected. Tolerance to parasite infection is the result of individuals investing in physiological mechanisms that limit fitness costs of infection without limiting parasite reproduction which has been proposed for other lizard malaria systems (Rausher, 2001; Bonneaud et al., 2017).Third, BCI—our proxy for fitness-may be weakly related to fitness in A. gundlachi. A recent meta-analysis shows large variability in how body condition indices relate to fitness in many host-parasite systems (Sánchez et al., 2018). Still, the lack of evidence for Plasmodium virulence to other Caribbean lizards is consistent. The study of Plasmodium spp. virulence to Anolis sabanus in Saba, an island geographically close to Puerto Rico, found no evidence of virulence looking at a wide array of variables which supports our results (Schall and Staats, 2002).

Overall, higher degrees of WIV in infected individuals might be explained by multiple types of interactions between A. gundlachi and Plasmodium. In humans, Plasmodium infection induces structural and functional changes in erythrocyte traits such as loss of discoid shape, increased membrane rigidity, elevated permeability, reduced deformability, and increased adhesiveness (Cooke et al., 2001). The higher WIV erythrocyte size variation in infected A.gundlachi may also be explained by induced erythropoiesis - erythrocyte production. Plasmodium infection results in the global destruction of erythrocytes and consequently the lizard may respond increasing the production of immature erythrocytes in the hemopoietic bone marrow (Zapata et al., 1981; Zivot et al., 2018). This process may lead to higher size variability in erythrocyte size in infected lizards compared to their non-infected counterparts. Higher numbers of immature erythrocytes have been found at weak and moderate degrees of parasitemia in a variety of Plasmodium lizard hosts including Sceloporus occidentalis, Agama agama and Anolis gingivinus (Schall, 1990, 1992). Still, we restricted our analysis to adult erythrocytes. Therefore, any observed effect of increased erythropoiesis in our system is not due to an increase in immature cells, but the residual effect on adult cells. While increased erythropoiesis is a parsimonious explanation for the increased WIV in infected lizards, WIV in animals could be the result of other complex interacting processes such as organ-level developmental plasticity, diet, reproductive state, metabolic rate, or genetic relatedness.

The study of the drivers and consequences of WIV is an emerging sub field in ecology and evolution (Herrera et al., 2015). Most of the evidence supporting the role of WIV comes from plant systems (Herrera, 2009). The apparent lack of obvious reiterative structures in animal systems limits our ability to generalize this theoretical framework. This study aims to contribute to the expansion of the study of WIV in animal 
systems by testing the role of erythrocytes as a reiterative internal structure. While we found a clear relationship between WIV in erythrocyte size and parasite infection status, our results showed no clear evidence that this WIV has an adaptive role. Likely the increase in WIV is a consequence of the host's response to infection. Still, the potential adaptive role of WIV in animal systems remains a wide and unexplored area of research. The mere presence of WIV on any system can have profound implications on sampling techniques and experimental designs used to study it. We hope our study encourages additional studies in other systems.

\section{CONCLUSIONS}

This is one of the few studies aimed to understand the drivers and consequences of within-individual variation in animal systems. We tested for the role of variation in erythrocyte size as an internal reiterative component potentially mediating interactions with natural enemies in a Caribbean lizard-malaria system. While we found a clear relationship between WIV and infection status, the lack of evidence of changes in body condition suggests that erythrocyte size variation is likely a consequence of infection and not necessarily adaptive.

\section{ACKNOWLEDGMENTS}

We would like to thank Elizabeth Evans, Gary Gervais, Wilson E. Lozano, Dr. Luisa Otero, Dr. María E. Pérez, Ana C. Medina, Ana Trujillo, Dr. Ethan P. White, Dr. Jos Schall, and Dr. Hao Ye for all their invaluable help. "This material is based upon work supported by the National Science Foundation Graduate Research Fellowship under Grant No. DGE-1315138 and DGE-1842473"

\section{REFERENCES}

Acevedo, M. A., Dillemuth, F. P., Flick, A. J., Faldyn, M. J., and Elderd, B. D. (2019). Virulence-driven trade-offs in disease transmission: A meta-analysis. Evolution, 73(4):636-647.

Allison, A. C. (1954). Protection afforded by sickle-cell trait against subtertian malarial infection. British medical journal, 1(4857):290.

Anderson, P. and Agrell, J. (2005). Within-plant variation in induced defence in developing leaves of cotton plants. Oecologia, 144(3):427-434.

Ardia, D. (2005). Super size me: an experimental test of the factors affecting lipid content and the ability of residual body mass to predict lipid stores in nestling european starlings. Functional Ecology, 19(3):414-420.

Atkinson, C., Woods, K., Dusek, R. J., Sileo, L., and Iko, W. (1995). Wildlife disease and conservation in hawaii: pathogenicity of avian malaria (plasmodium relictum) in experimentally infected iiwi (vestiaria coccinea). Parasitology, 111(S1):S59-S69.

Austen, E., Forrest, J., and Weis, A. (2015). Within-plant variation in reproductive investment: consequences for selection on flowering time. Journal of Evolutionary Biology, 28(1):65-79.

Bee, M. A. (2004). Within-individual variation in bullfrog vocalizations: Implications for a vocally mediated social recognition system. The Journal of the Acoustical Society of America, 116(6):37703781.

Biernaskie, J. M., Cartar, R. V., and Hurly, T. A. (2002). Risk-averse inflorescence departure in hummingbirds and bumble bees: could plants benefit from variable nectar volumes? Oikos, 98(1):98-104.

Bonneaud, C., Sepil, I., Wilfert, L., and Calsbeek, R. (2017). Plasmodium infections in natural populations of anolis sagrei reflect tolerance rather than susceptibility. Integrative and Comparative Biology, 57(2):352-361.

Castro, E. N., Lomonte, B., del Carmen Gutiérrez, M., Alagón, A., and Gutiérrez, J. M. (2013). Intraspecies variation in the venom of the rattlesnake crotalus simus from mexico: Different expression of crotoxin results in highly variable toxicity in the venoms of three subspecies. Journal of Proteomics, $87: 103-121$.

Cohen, J. (1992). A power primer. Psychological bulletin, 112(1):155.

Cooke, B. M., Mohandas, N., and Coppel, R. L. (2001). The malaria-infected red blood cell: structural and functional changes. Advances in Parasitology, 50:1-86.

Cox, R. M. and Calsbeek, R. (2015). Survival of the fattest? indices of body condition do not predict viability in the brown anole (a nolis sagrei). Functional Ecology, 29(3):404-413. 
Donnelly, S. M. and Kramer, A. (1999). Testing for multiple species in fossil samples: an evaluation and comparison of tests for equal relative variation. American Journal of Physical Anthropology: The Official Publication of the American Association of Physical Anthropologists, 108(4):507-529.

Haukioja, E. and Niemelä, P. (1979). Birch leaves as a resource for herbivores: seasonal occurrence of increased resistance in foliage after mechanical damage of adjacent leaves. Oecologia, 39(2):151-159.

Herrera, C. M. (2009). Multiplicity in unity. University of Chicago Press.

Herrera, C. M., Medrano, M., and Bazaga, P. (2015). Continuous within-plant variation as a source of intraspecific functional diversity: Patterns, magnitude, and genetic correlates of leaf variability in helleborus foetidus (ranunculaceae). American Journal of Botany, 102(2):225-232.

Jakob, E. M., Marshall, S. D., and Uetz, G. W. (1996). Estimating fitness: a comparison of body condition indices. Oikos, pages 61-67.

Langkilde, T. and Shine, R. (2006). How much stress do researchers inflict on their study animals? a case study using a scincid lizard, eulamprus heatwolei. Journal of Experimental Biology, 209(6):1035-1043.

Matthews, A. E., Ellis, V. A., Hanson, A. A., Roberts, J. R., Ricklefs, R. E., and Collins, M. D. (2016). Avian haemosporidian prevalence and its relationship to host life histories in eastern tennessee. Journal of Ornithology, 157(2):533-548.

Nakayama, S., Laskowski, K. L., Klefoth, T., and Arlinghaus, R. (2016). Between-and within-individual variation in activity increases with water temperature in wild perch. Behavioral Ecology, page arw090.

Otero, L., Schall, J. J., Cruz, V., Aaltonen, K., and Acevedo, M. A. (2019). The drivers and consequences of unstable plasmodium dynamics: a long-term study of three malaria parasite species infecting a tropical lizard. Parasitology, 146(4):453-461.

Pearson, A. R. (2000). Body condition of a puerto rican anole, anolis gundlachi: Effect of a malaria parasite and weather variation. Journal of Herpetology, 34(3):489-491.

Perkins, S. L. and Schall, J. (2002). A molecular phylogeny of malarial parasites recovered from cytochrome b gene sequences. Journal of Parasitology, 88(5):972-978.

Rausher, M. D. (2001). Co-evolution and plant resistance to natural enemies. Nature, 411(6839):857-864. Reagan, D. P. and Waide, R. B. (1996). The food web of a tropical rain forest. University of Chicago Press.

Real, L. A. and Rathcke, B. J. (1991). Individual variation in nectar production and its effect on fitness in kalmia latifolia. Ecology, 72(1):149-155.

Roth, E. F., Friedman, M., Ueda, Y., Tellez, I., Trager, W., and Nagel, R. L. (1978). Sickling rates of human as red cells infected in vitro with plasmodium falciparum malaria. Science, 202(4368):650-652.

Sánchez, C. A., Becker, D. J., Teitelbaum, C. S., Barriga, P., Brown, L. M., Majewska, A. A., Hall, R. J., and Altizer, S. (2018). On the relationship between body condition and parasite infection in wildlife: a review and meta-analysis. Ecology letters, 21(12):1869-1884.

Schall, J. (1990). Virulence of lizard malaria: the evolutionary ecology of an ancient parasite-host association. Parasitology, 100(S1):S35-S52.

Schall, J., Pearson, A. R., and Perkins, S. L. (2000). Prevalence of malaria parasites (Plasmodium floridense and Plasmodium azurophilum) infecting a puerto rican lizard (Anolis gundlachi): a nine-year study. Journal of Parasitology, 86(3):511-515.

Schall, J. and Staats, C. M. (2002). Virulence of lizard malaria: three species of plasmodium infecting anolis sabanus, the endemic anole of saba, netherlands antilles. Copeia, 2002(1):39-43.

Schall, J. J. (1992). Parasite-mediated competition in anolis lizards. Oecologia, 92(1):58-64.

Schall, J. J. (1996). Malarial parasites of lizards: diversity and ecology. Advances in Parasitology, 37:255-333.

Schall, J. J. and Vogt, S. P. (1993). Distribution of malaria in anolis lizards of the luquillo forest, puerto rico: implications for host community ecology. Biotropica, pages 229-235.

Schneider, C. A., Rasband, W. S., and Eliceiri, K. W. (2012). Nih image to imagej: 25 years of image analysis. Nature Methods, 9(7):671-675.

Schulte-Hostedde, A. I., Zinner, B., Millar, J. S., and Hickling, G. J. (2005). Restitution of mass-size residuals: validating body condition indices. Ecology, 86(1):155-163.

Shafir, S. (2000). Risk-sensitive foraging: the effect of relative variability. Oikos, 88(3):663-669.

Shimada, T., Takahashi, A., Shibata, M., and Yagihashi, T. (2015). Effects of within-plant variability in seed weight and tannin content on foraging behaviour of seed consumers. Functional Ecology, 29(12):1513-1521. 
Sion, G., Watson, M., and Bouskila, A. (2021). Measuring body condition of lizards: a comparison between non-invasive dual-energy $\mathrm{x}$-ray absorptiometry, chemical fat extraction and calculated indices. Frontiers in Zoology, 18(1):1-9.

Stahl, P. D., Christensen, M., and Williams, S. (1990). Population variation in the mycorrhizal fungus glomus mosseae: uniform garden experiments. Mycological Research, 94(8):1070-1076.

Tanner, J. C. and Bee, M. A. (2019). Within-individual variation in sexual displays: signal or noise? Behavioral Ecology, 30(1):80-91.

Telford, S. R. (2009). Hemoparasites of the Reptilia. CRC Press.

Whitham, T. G. (1983). Host manipulation of parasites: within-plant variation as a defense against rapidly evolving pests. Variable plants and herbivores in natural and managed systems. Academic Press New York, USA.

Whitham, T. G. and Slobodchikoff, C. (1981). Evolution by individuals, plant-herbivore interactions, and mosaics of genetic variability: the adaptive significance of somatic mutations in plants. Oecologia, 49(3):287-292.

Wilder, S., Raubenheimer, D., and Simpson, S. (2016). Moving beyond body condition indices as an estimate of fitness in ecological and evolutionary studies. Functional Ecology, 30(2):108-115.

Zapata, A., Leceta, J., and Villena, A. (1981). Reptilian bone marrow. an ultrastructural study in the spanish lizard, lacerta hispanica. Journal of morphology, 168(2):137-149.

Zivot, A., Jeffrey, M., Anupama, N., and Lionel, B. (2018). Erythropoiesis: insights into pathophysiology and treatments in 2017. Molecular Medicine, 24(1):1-15. 


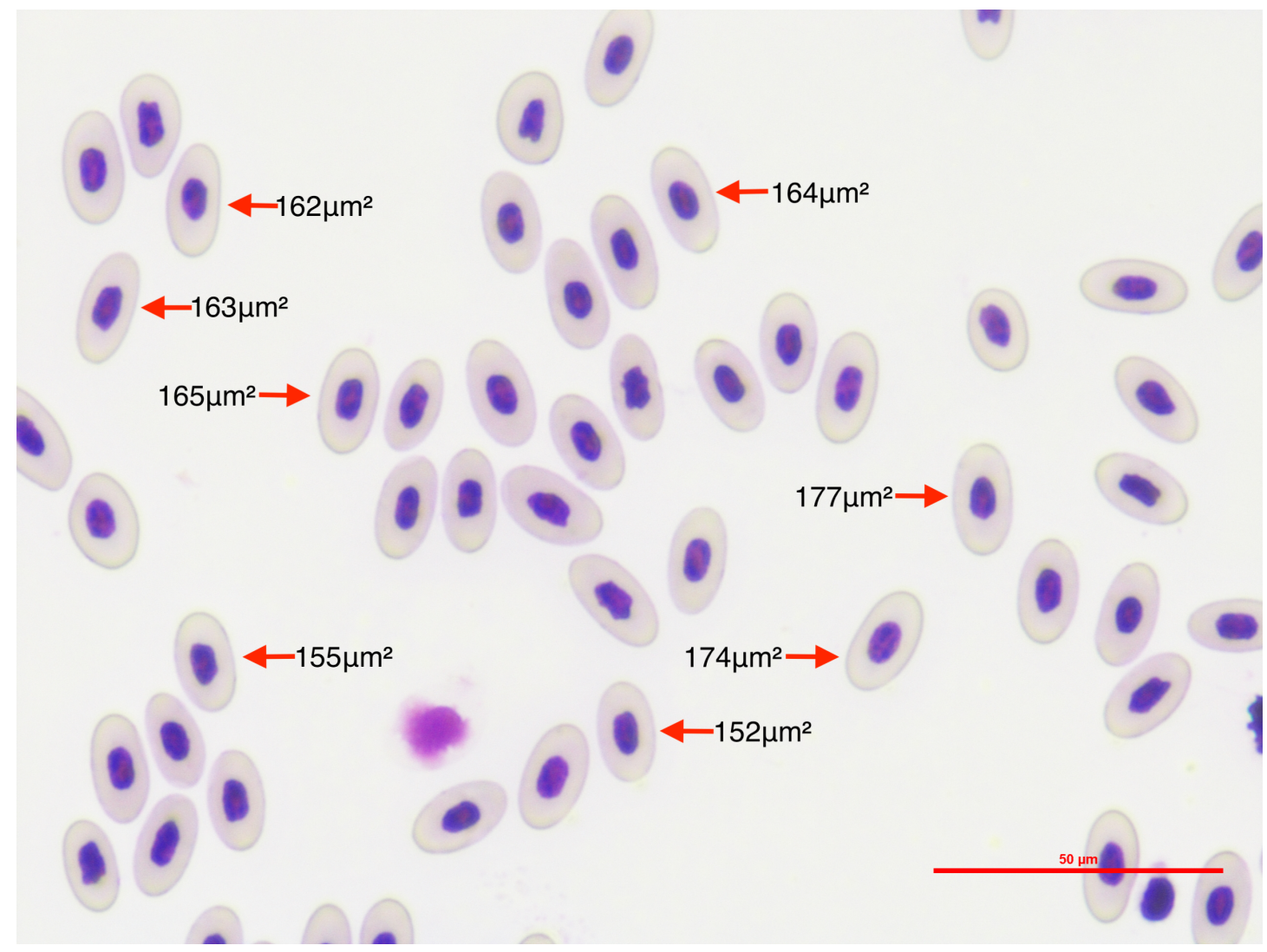

Figure 1. To quantify the within-individual variation in lizard erythrocyte size we photographed thin blood smear optical fields of $P$. azurophilum infected and non-infected A.gundlachi individuals. The figure shows an example of photographed optical field of a thin blood smear sample at a 40x magnification of A.gundlachi individual. The numbers represent erythrocyte areas $\left(\mu \mathrm{m}^{2}\right)$. We measured mature erythrocytes and calculated the coefficient of variation of cell size per lizard. 

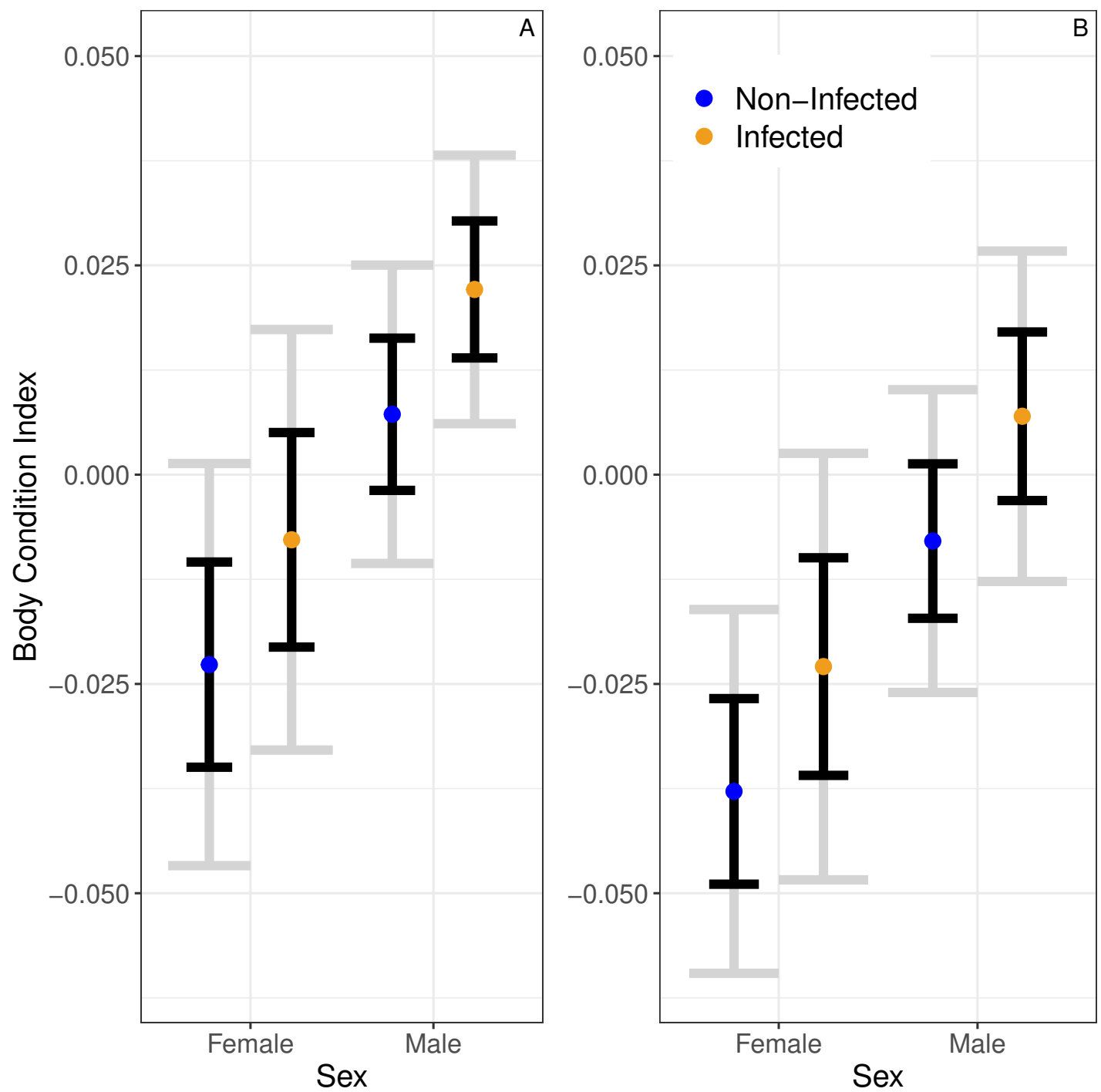

Figure 2. The figure shows the predicted partial relationships between infection status and body condition index (BCI) for female and male A.gundlachi during the A) 2015 summer B) and 2016 winter seasons. Grey error bars represent $95 \% \mathrm{CI}$ and black error bars represent one standard error. 

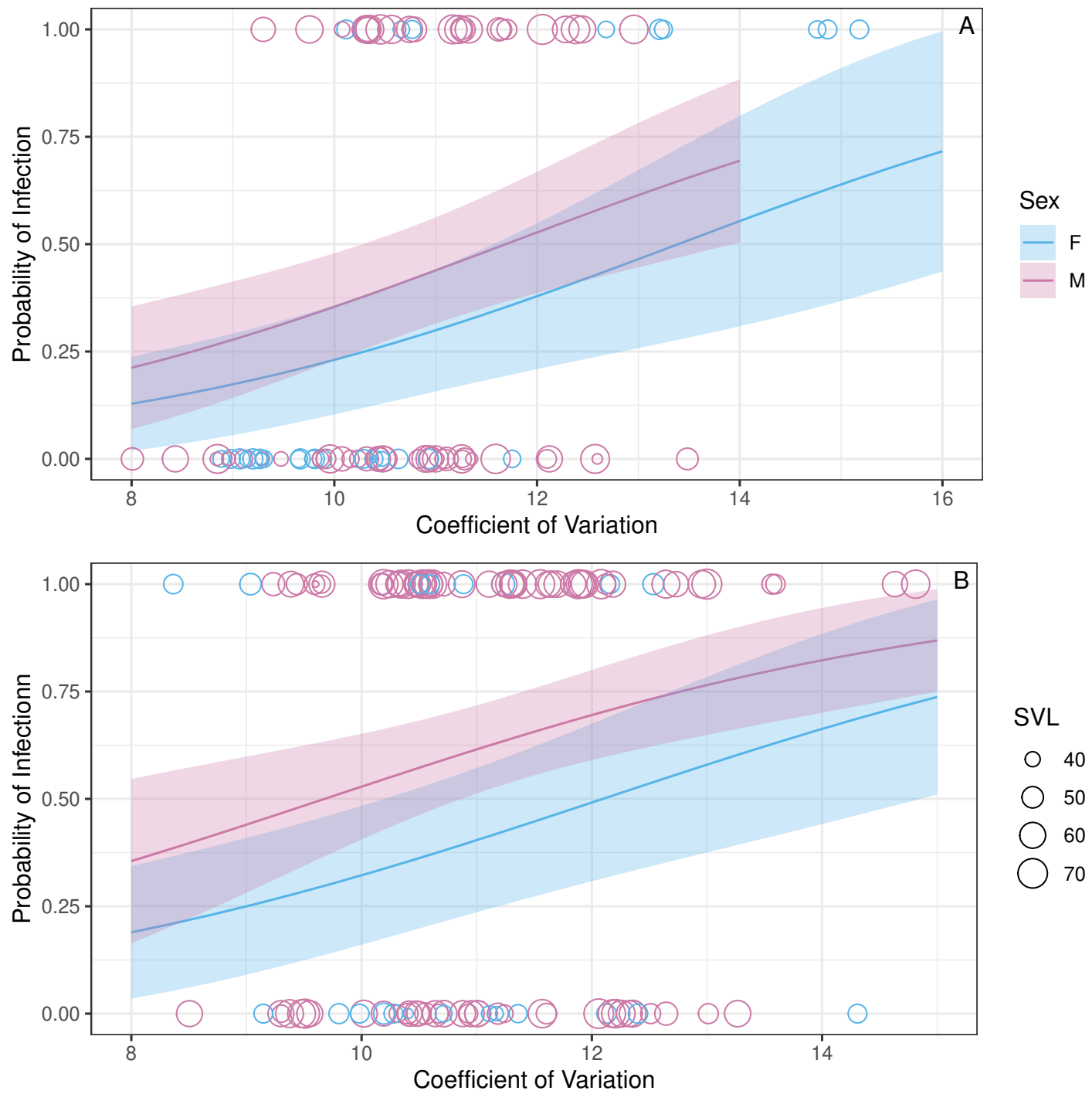

Figure 3. The model predicts that the probability of infection increases with increasing WIV quantified here as CV in erythrocyte size. The figure shows partial predictions for male and female $A$. gundlachi during the a) 2015 summer season and b) 2016 winter season. The size of the points represents relative SVL and their color infection status. 

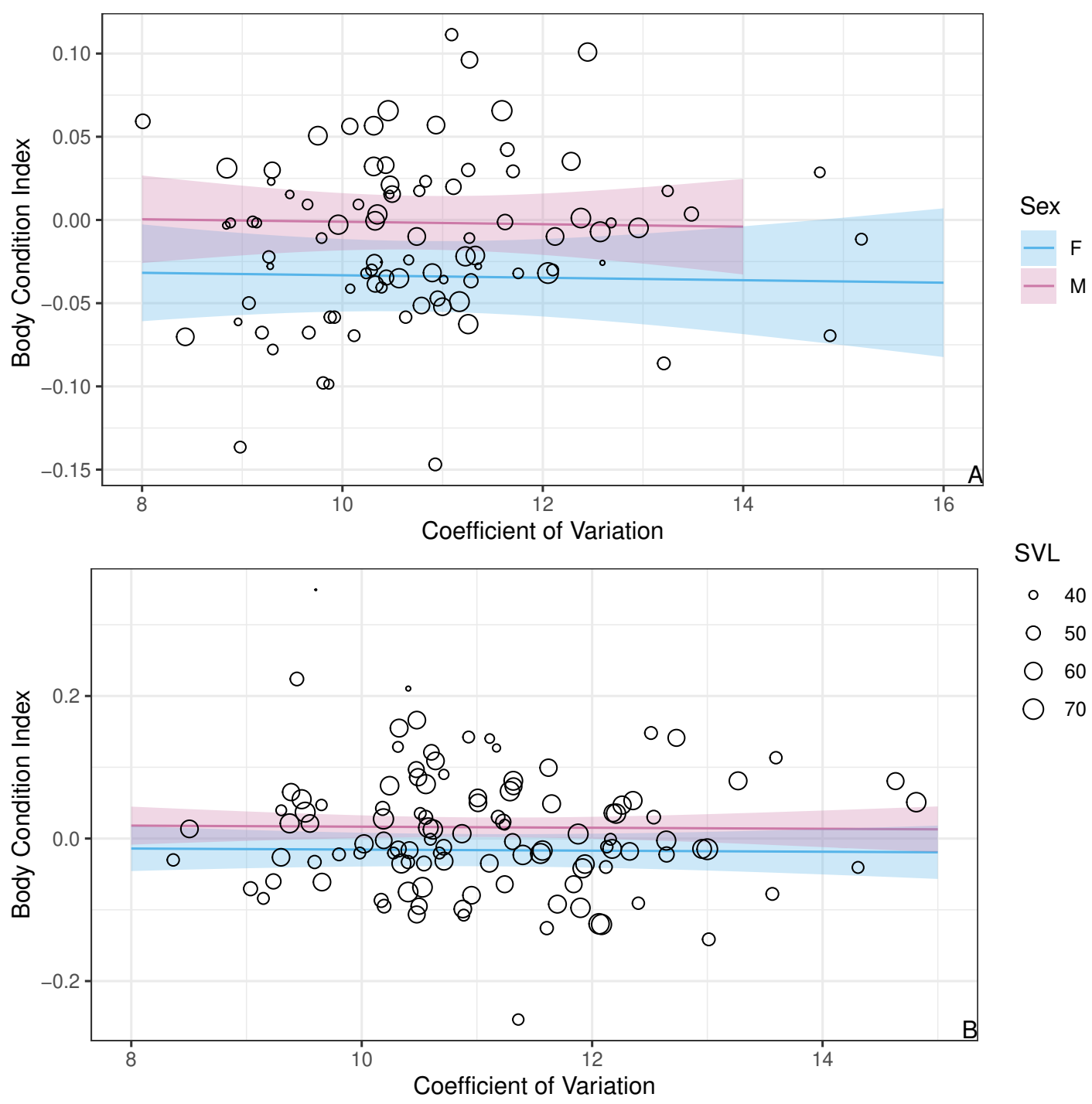

Figure 4. The model predicts no significant relationship between $\mathrm{CV}$ of erythrocyte size and BCI. The figure shows partial predictions for female and male A.gundlachi during the a) 2015 summer season and b) 2016 winter season. The size of the points represents relative SVL. 